\title{
KÖRPER UND TEMPERATUREN: FIGURATIONEN DES KOLONIALEN BEGEHRENS IN DER DEUTSCHSPRACHIGEN GEGENWARTSLITERATUR
}

\begin{abstract}
Body and Temperature: Configurations of Colonial Desire in Contemporary German Literature
The central argument of this essay is gathered from two historical novels published around same time and revolving around the same historical figure August Engelhardt. High temperature, in particular the tropical heat finds its metaphorical expression in the sun and then the fever as two prototypical colonial phantasies and/or fears and weaves the narrative around the temperature driven intricacies. Marc Buhls Das Paradies des August Engelhardt (2011) and Christian Krachts Imperium (2012) form the primary sources for the discussion within the topic of colonial desire with reference to the temperature as a significant indicator of either appreciation or devaluation underlining the colonial mentality. My paper concentrates on the overlapping sites within German speaking literature from post-colonial perspective. Along with the works of Marc Buhl and Christian Kracht, I cursorily touch upon Ilija Trojanows novel Der Weltensammler (2006), since it deals with the figure of a historical British colonial officer, Richard Francis Burton. The common thread running through all three works is not only the fact that they deal with scurrile biographies from the pages of colonial history in India and Africa, but that they develop the narrative on another common indicator - the Tropes and their Temperatures.
\end{abstract}

KEYWORDS: Temperature, tropes, sun-worship, nudity, German colonies, post-colonial literature

In seinem Buch Ein Bild von Afrika. Rassismus in Conrads ,Herz der Finsternis “ wirft Chinua Achebe Conrad vor, er benutze Afrika nur als „Kulisse und Hintergrund, der den Afrikaner als menschlichen Faktor eliminiert. Afrika ist ein metaphysisches Schlachtfeld ohne jede erkennbare Menschlichkeit, das der umherziehende Europäer auf eigene Gefahr betritt. “1 Eine ähnliche Funktion als Kulisse und Hintergrund wurde zahlreichen Texten deutscher Autor/innen über Indien attestiert, beson-

\footnotetext{
${ }^{1}$ Chinua Achebe: Ein Bild von Afrika. Rassismus in Conrads ,Herz der Finsternis “. Deutsch von Thomas Brückner und Wulf Teichmann. Berlin 2000, S. 26.
} 
ders der Indomanie der deutschen Schriftsteller und Philosophen im 19. Jahrhundert. Was die exotisierende Seite der Faszination, aber auch des Schreckens ${ }^{2}$, ausmachte, waren vornehmlich solche Phänomene wie die warmen Temperaturen, die paradiesähnlichen Naturzustände, die ,wilde' Natur und die ,natürlichen' Wilden. Innerhalb der Kolonialismus-Debatte gibt es mehrere literarische Beispiele aus dem deutschsprachigen Raum, die den ,postkolonialen Blick ${ }^{\star 33}$ schärfen oder schärfen sollten.

Im Fokus meines Beitrags stehen zwei innerhalb eines Jahres erschienene historische Romane mit derselben realhistorischen Figur, August Engelhardt, in denen die Sonne und das Fieber als zwei prototypische Kolonialphantasien bzw. Schrecken im Mittelpunkt stehen. Marc Buhls Das Paradies des August Engelhardt (2011) ${ }^{4}$ und Christian Krachts Imperium (2012) ${ }^{5}$ bilden die Grundlage der Diskussion um den Topos des kolonialen Begehrens in Anlehnung an die Temperaturen als ein aufwertendes bzw. abwertendes Prinzip der kolonialen Denkweise. Es stellen sich die Fragen: Warum greift man historische Fakten als literarische Motive wieder auf, besonders wenn sie die Kolonialgeschichte eines Landes umfassen? Inwiefern ist dieses Phänomen des Wiedersehens, eines bewusst erweckten Déjà-vu mit historischen Fakten, als gegenwärtiger Kolonialdiskurs zu verstehen? Welche anderen Diskurse bedient der Versuch, Geschichte durch Literatur darzustellen? Mein Beitrag richtet sein Augenmerk auf die Überschneidungen von Literatur und Geschichte in der deutschsprachigen Gegenwartsliteratur aus postkolonialem Blick. Zusammen mit den Romanen von Buhl und Kracht wird auch Ilija Trojanows Roman Der Weltensammler (2006) ${ }^{6}$ - allerdings nur kursorisch - erwähnt, da auch er in der historischen Biographie eines Kolonialoffiziers, hier des Briten Richard Francis Burton, seine Erzählinspiration findet. Was die drei Werke verbindet, sind nicht nur die skurrilen Biographien der jeweiligen realhistorischen Figuren, sondern auch die Tropen und deren Temperaturen.

Die Biographie August Engelhardts (1875-1919) hängt unmittelbar mit der kolonialen Vergangenheit Deutschlands zusammen. 1884 wurde der Deutsche Kolonialverein gegründet und das Deutsche Reich erhielt seine ersten Kolonien, die sog. Schutzgebiete, in Afrika. Zum deutschen Kolonialbesitz gehörten Deutsch-Südwestafrika, Togo, Kamerun, Deutsch-Ostafrika und Deutsch-Neuguinea. In der Südsee umfassten die Kolonien eine Reihe von Inselgruppen im Pazifik, die von Samoa über die Salomonen und die Marshallinseln, die Karolinen und die Marianen, bis nach Neuguinea reichten. Es gab in Deutschland zahlreiche Veröffentlichungen, die die Begegnung mit der Südsee aus ökonomischer, geographischer, naturwissen-

\footnotetext{
${ }^{2}$ Vgl. Rolf-Peter Janz (Hrsg.): Faszination und Schrecken des Fremden. Frankfurt a. M. 2001.

${ }^{3}$ Vgl. Paul Michael Lützeler (Hrsg.): Der postkoloniale Blick. Deutsche Schriftsteller berichten aus der Dritten Welt. Frankfurt a. M. 1997.

${ }^{4}$ Marc Buhl: Das Paradies des August Engelhardt. Roman. Frankfurt a. M. 2011.

${ }^{5}$ Christian Kracht: Imperium. Roman. Köln 2012.

${ }^{6}$ Ilija Trojanow: Der Weltensammler. Roman. München 2006.
} 
schaftlicher und ethnographischer Sicht erschließen wollten. ${ }^{7}$ Die Zahl der Studien, die sich mit den verschiedensten Genres wie Reiseberichten, wissenschaftlichen Abhandlungen, Memoiren, Missionsberichten sowie fiktionaler Literatur beschäftigen, nimmt zu und wird immer häufiger durch den postkolonialen Blick begleitet. Gabriele Dürbecks Analyse des Ozeanismus liegen drei Kriterien zugrunde, die auch bei der Betrachtung von neueren literarischen Beispielen von Bedeutung sind. Die Kriterien lauten: erstens - die implizite oder explizite Bestätigung des kolonialen Diskurses, zweitens - die Mehrdimensionalität und häufige Ambivalenz von Positionen und drittens - die Frage, ob das Werk nur exotistische Darstellungen zu bieten hat oder auch europa- und missionskritische Aussagen trifft. ${ }^{8}$ Meine Analyse der ausgewählten Werke wird auf diese Kriterien Bezug nehmen.

Warum hat August Engelhardt die Autoren wie Marc Buhl und Christian Kracht in seinen Bann gezogen? Er war ein Apotheker aus Nürnberg, der 1902 die Insel Kabakon in dem Schutzgebiet Deutsch-Neuguinea von der damals reichsten Frau Ozeaniens, der Halbsamoanerin „Queen Emma“, erworben hatte. Er gründete dort seinen Sonnenorden, der bis zu 30 Mitgliedern aus Deutschland angezogen haben soll. Sein Ziel war, die Kokosplantagen auf der Insel zu bewirtschaften und vor allem eine radikale ,Lebens-re-form' zu praktizieren. Diese bestand aus Vegetarismus bzw. Kokovorismus, Freikörperkultur und einer extremen Sonnenzugewandtheit. Sein Freund, der Schriftsteller August Bethmann, und seine Verlobte Anna Schwab gehörten zu den wichtigsten Figuren im Leben Engelhardts. Die Freunde haben auf der Insel einige Zeit verbracht, am Ende soll u. a. die Eifersucht zu einer Kluft zwischen Engelhardt und Bethmann geführt haben. Nach dem Versailler Vertrag von 1919 musste Deutschland die Inseln des Bismarck-Archipels samt Kabakon an Australien abtreten. Engelhardt starb dort im gleichen Jahr.

Buhl und Kracht gehen mit der Biographie des „ersten Hippie[s] aus dem Kaiserreich ${ }^{\text {“9 }}$ unterschiedlich um. Interessant ist im Kontext des Temperaturendiskurses die Herausarbeitung des Fremdheitsdiskurses aus dem Kolonialdiskurs und die engmaschige Verknüpfung zwischen den Begriffen Aneignung, Enteignung und Entfremdung. Es lassen sich zwei auffallende Topoi finden, die sich gleichzeitig als aufwertende bzw. abwertende Anhaltspunkte zur Auseinandersetzung mit dem Kolonialdiskurs anbieten. Es sind die Topoi der Sonnenverehrung/-anbetung und die Angst vor dem Tropenfieber. Engelhardts Begeisterung für die Sonne und deren göttliche Kräfte lässt an den die Tropen und den Orient exotisierenden Blick der deutschen Romantiker zurückdenken. Engelhardt wird zu einem Sonnenfanatiker,

\footnotetext{
${ }^{7}$ Vgl. Gabriele Dürbeck: Stereotype Paradiese. Ozeanismus in der deutschen Südseeliteratur. 1815-1914. Tübingen 2007.

${ }^{8}$ Vgl. ebd., S. 7.

${ }^{9}$ Zitiert nach: Catherine Repussard: Ein bisschen Südsee und ein gutes Maß Lebensreform: Das Rezept für das beginnende 21. Jahrhundert? Marc Buhls „Paradies des August Engelhardt“ (2011) und Christian Krachts ,Imperium“ (2012). In: Recherches Germaniques 42 (2012), S. 77-98, hier S. 78.
} 
der nackt das Sonnenlicht als eine Heilquelle einnehmen bzw. zu sich nehmen will. Er macht die Solarenergie quasi zu einem Nahrungsmittel, indem er sie ,trinkt ${ }^{\star}$. Die Sonnenhuldigung hängt ,hautnah“ mit seiner anderen Vorstellung von einer ,Lebensre-form', der Nacktheit, zusammen, die neben der Sonnenhuldigung und dem Kokovorismus, zu Engelhardts Welt- und Lebensreformrezept gehört. Er will Pflanzer werden, ,doch nicht aus Profitgier, sondern aus zutiefst empfundenem Glauben, er könne Kraft seiner großen Idee die Welt, die ihn feindlich, dumm und grausam dünkte, für immer verändern. “"10

Die drei Rezeptzutaten - die Sonnenhuldigung, die Nacktheit und der Kokovorismus - werden zu einer Schnittstelle, an der der Kolonialdiskurs in den beiden Romanen seine unterschiedlichen Konturen gewinnt. Was für Engelhardt in Krachts Roman „Sirenenrufe des Paradieses“11 sind, sind für die Vertreter des deutschen Kolonialreichs, wie den Gouverneur Hahl, Abgründe der Wildnis und Barbarei sowie bedrohliche Fallen, die eigene Autorität in den Kolonien zu verlieren. Für die Eingeborenen sind Engelhardts Nacktheit und seine Kokosverehrung ein Indiz dafür, dass er ,anders' ist und ihren sonst üblichen kannibalischen Ritualen nicht zum Opfer fallen darf. Bei Buhl wird die ambivalente Positionierung der Figur Engelhardts auffällig. Durch seine Europamüdigkeit und Zivilisationsfeindseligkeit wirkt er auch kolonialkritisch und behält gleichzeitig bis zu einem gewissen Grad sein Herrscherprofil des Kolonisators.

Im Gegensatz zu Kracht räumt Buhl den Eingeborenen eine selbstständige Erzählperspektive ein. Wie in Trojanows Roman Der Weltensammler lässt sich auch in dem Werk von Buhl eine polyphone Erzählstruktur beobachten. Kabua, der Tolai Häuptling, stellt etwas Zentrales fest. Engelhardt, so bemerkt er, sei kein Weißer, nicht nur, weil bei ihm die Kleider fehlen, sondern auch deswegen, weil er kein Ziel habe. Seine Erfahrung mit den Weißen hat ihn gelehrt, dass „die Weißen immer ein Ziel [haben], und wenn es keines gibt, dann schaffen sie es, und können sie keines schaffen, werden sie krank." ${ }^{\text {"12 }}$ Der langhaarige Vertreter der Freikörperkultur wird zu einem Störfaktor für die Kolonialherren auf der Insel. Er hat für das Kolonialreich keinen direkten Gebrauchswert, wie ihn ein Tischler, ein Arzt, ein Pflanzer oder eine Krankenschwester haben könnten. Die Auseinandersetzungen zwischen den Vertretern von den Hauptagenten der kolonialen Mechanismen, nämlich der Religion (Pater Joseph) und der Verwaltung (Gouverneur Hahl) sowie Engelhardt werden zu Arenen der Anschauungskonflikte über Natur und Kultur, koloniale Machtausübung und individuelle Lebensphilosophie. Es erscheint besonders interessant, wie die Sonne in diesem Roman zu einem heiß umkämpften Kriegsschauplatz der Ideologien wird. Gouverneur Hahl ist fest überzeugt, dass die Verehrung der

\footnotetext{
${ }^{10}$ Kracht: Imperium, S. 19.

${ }^{11}$ Ebd., S. 20.

${ }^{12}$ Buhl: Das Paradies des August Engelhardt, S. 18.
} 
Sonne keine Würde verleihen kann: „die schien hier sowieso viel zu viel und brannte einem das Hirn weg, wenn man nicht aufpasste, [stattdessen brauchte man] Anstand, Ordnung und Arbeit, daran mangelte es ....".13

Das koloniale Begehren gehört zu den festen Bestandteilen der Lektüren, die die Kolonien als Kulisse und Hintergrund gebrauchen. Die verführerischen Aspekte der fremden Welt haben auch mit den Spiegelungsmechanismen zu tun. ${ }^{14}$ Herbert Uerlings gebraucht den Begriff des Primitivismus, um die Beziehung zwischen zwei Kulturen zu erläutern. Er definiert den Primitivismus als

[...] eines der Modelle, nach denen das Verhältnis zwischen eigener und fremder Kultur gedacht wird. Dabei denkt sich die eine, die eigene Kultur als eine komplexe und dynamische Kultur, und sie denkt sich die andere, die fremde Kultur als das genaue Gegenteil, nämlich als einfach und statisch, also als (mehr oder weniger) geschichtslos. Das ist der Grundgedanke: die Unterscheidung zwischen ,einfach' und ,komplex' und die Anordnung auf einer Zeitachse: Vergangenheit - Gegenwart. ${ }^{15}$

Die Spiegelungsmechanismen sind im Prinzip Wertungsparameter, durch die die fremde Kultur entweder abgewertet oder aufgewertet wird. Wird das ,Primitive“ abgewertet, dann versichert man sich seiner eigenen ,besseren' Position, wohingegen man bei der Aufwertung der fremden Kultur selbstkritisch agiert. Uerlings versteht diese Mechanismen als Grundbestandteile des Primitivismus in Literatur und bildender Kunst. Sie ,gehör[en] zu den wirkungsmächtigsten und folgenreichsten Modellen der Erklärung und des Verstehens fremder Kulturen.“"16

Die kolonialen Phantasien haben zum größten Teil sexuellen Charakter. Bei Kracht befinden sich an Bord des Schiffes namens Prinz Waldemar dickleibige Pflanzer, die „schmatzend von barbusigen dunkelbraunen Negermädchen träumen“. ${ }^{17}$ Sogar den Gegenständen auf dem Schiff wird eine geschlechtliche Funktion zugewiesen. Hartmut Otto, ein Pflanzer und Paradiesvögelhändler, erklärt Engelhardt, dass der Deckstuhl wegen seiner nach vorne ausgestreckten Beine „Bombay Fornicator“ heißt. Engelhardt reist bei Kracht sowie bei Buhl über Indien zu den Südseeinseln.

Wie die Geschichte der Entwicklung des Kolonialismus nachgewiesen hat, gibt es eine enge Beziehung zwischen der Agri-Kultur und dem Projekt, eine Gegend aus kolonialen Interessen zu bewirtschaften, sie fruchtbar zu machen (Agrar-Wirtschaft).

\footnotetext{
${ }^{13}$ Ebd., S. 36.

${ }^{14}$ Herbert Uerlings bespricht diese Spiegelungsmechanismen mit Bezug auf den Primitivismus in Literatur und bildenden Künsten und weist auf den zerbrochenen Spiegel, Europas vis-à-vis der Kulturen der Eingeborenen, hin. Vgl. Herbert Uerlings: Verkehrte Welten. Primitivismus in Literatur und Kunst der Klassischen Moderne. In: Tobias L. Kienlin (Hrsg.): Fremdheit - Perspektiven auf das Andere. Bonn 2015 (= Kölner Beiträge zu Archäologie und Kulturwissenschaften, Bd. 264), S. 9-24 [Kursive im Original].

${ }^{15}$ Ebd., S. 9.

${ }^{16}$ Ebd.

${ }^{17}$ Kracht: Imperium, S. 13.
} 
Dies führt auch zu der oft angewendeten Analogie zwischen der weiblichen Sexualität und den Kolonien. Der Analogie liegt die Annahme zugrunde, dass die in den Kolonien verfügbaren indigenen Frauen nicht nur der sexuellen Lust der Kolonialherren unterliegen, sondern dass die Macht über die Frauen gleichzeitig auch die Macht über das Land symbolisiert. Deshalb ist es entscheidend zu fragen, ob der freie Geschlechtsverkehr zwischen weißen Männern und indigenen Frauen stattfindet, oder ob weiße Frauen auch indigene Männer als sexuelle Partner auswählen? Im Falle der weißen Frauen, die nichts gegen geschlechtliche Beziehungen mit Kabua haben, werden diese von den autoritätsbewussten Herren nicht nur als empörend und verschmähend wahrgenommen, sondern auch als ein klares Signal der Sittlichkeitsschändung in Europa, falls dieses Verhalten durch die Kolonialpresse in Deutschland bekannt werden sollte. Engelhardt ist aber von seiner Idee, die Welt zu verändern und mit radikal neuen Aposteln des Sonnenordens zu infiltrieren, dermaßen besessen, dass ihm Kalauer geschlechtlicher Natur suspekt werden. Er selbst interessiert sich für keine Frauen auf der Insel, weder für Eingeborene noch für Europäerinnen. Seine verdrängten Liebesgefühle für Anna Schwab, die Verlobte seines Freundes Bethmann, die ihn auf Kabakon besucht, kommen immer wieder zum Vorschein und suchen ihn sogar in seinen Fieberträumen heim.

Die Wahrnehmung der kolonialen Welt oszilliert zwischen der binär imaginierten Konstruktion der Aufwertung und Abwertung. Ich möchte hier argumentieren, dass die heißen Temperaturen je nach der Perspektive über verschiedene diskursive Funktionen verfügen. Die glühende Sonne und das hohe Tropenfieber haben ähnliche Temperaturkonfigurationen, jedoch mit einer völlig unterschiedlicher interpretativer Wirkung. Die Sonnenverehrung wird von Engelhardt als ein Topos zur Aufwertung der sonst ,primitiven' Insel geschaffen und der Fieberschrecken wird zum Gegenpol der Dämonisierung der Inselerfahrung. In diesem Sinne werden - dem postkolonialen Diskurs entsprechend - klassische Gegensatzpaare konstruiert - die Verliebtheit und der Hass, die Faszination und der Schrecken, die Freiheit und die Krankheit, kurzum das ,edle“ Wilde und das ,nicht so edle“ Wilde. Einerseits verherrlicht Engelhardt das heilige Sonnenreich, andererseits diskutieren die anderen Europäer auf der Insel über die „destruktive Macht der Malaria““. ${ }^{18}$ Johannes Fabian geht in seiner Studie über Reiseberichte im deutschen Kolonialreich der besonderen Stellung des Tropenfiebers nach: „Fieber war eine Ideologie, ein Mythos, den man brauchte, um aus den tödlichen Gefahren der Afrikaforschung klug zu werden, eine Metapher, die Abläufen einen Sinn gab, welche sonst brutale Fakten geblieben wären.“19

Das Fieber wurde bei den Reisenden unabhängig von den psychosomatischen Zuständen wie Überanstrengung oder Müdigkeit verursacht, denn es konnte ebenso

\footnotetext{
${ }^{18}$ Ebd., S. 171.

${ }^{19}$ Johannes Fabian: Im Tropenfieber. Wissenschaft und Wahn in der Erforschung Zentralafrikas. München 2001, S. 91.
} 
durch übermäßige Aufregung, exzessiven Zorn und Ärger hervorgerufen werden. Das Malariafieber und der Tropenkoller als Aggressions- und Wutanfall waren oft vorkommende Krankheiten, die in fiktionalen und wissenschaftlichen Lektüren über die deutschen Kolonialexpeditionen dokumentiert worden sind. ${ }^{20}$ Ausdrücke wie das Reisefieber, das Fieber der Ungeduld, das Fieber des Liebesrausches usw. waren keine Seltenheit in diesen Texten und waren damals wörtlich gemeint. Fabian weist in diesem Zusammenhang auf einen interessanten Begriff hin - die Entdoppelung. Gemeint ist damit ein Zustand im hohen Fieber, in dem die Reisenden unter Halluzinationen gelitten haben sollen, so dass eine zusätzliche Person mit ihnen auf demselben Bett lag. Sobald es ihnen etwas besser ging, konnten sie sich bemühen, sich bewusst zu entdoppeln. ${ }^{21}$ Der Akt der Entdoppelung ist insofern wichtig, als er die doppelte Belastbarkeit, und in einigen Fällen auch die Hinfälligkeit der Europäer auf dem Schwarzen Kontinent, unterstrichen hat. Als Europäer mussten sie immer darauf achten, ihr überlegenes Profil als Forschungsreisende oder Pflanzer aufrechtzuerhalten.

Das Malariafieber (oder das Schwarzwasserfieber als eine Folgeerkrankung der Malaria) erhält bei Engelhardt und seinen Landsleuten eine große Symbolkraft. Das Fieber wird mehr als nur ein Instrument zur Dämonisierung der Insel und damit der kolonialen Welt. Der Fieberdiskurs verläuft darüber hinaus als Diskurs der Zivilisationskritik, indem die körperliche Krankheit mit etlichen sozialen Krankheiten in der europäischen Konsumgesellschaft in Verbindung gebracht wird. Das Malariafieber in Afrika übernimmt eine Warnfunktion für die ,Zerseuchung' der Seele durch den Kolonialwahn und die Konsumsucht der Europäer. Für Engelhardt ist zum Beispiel das Malariafieber

[...] eben der Preis, den man hier zu zahlen habe. Im Fränkischen gebe es wenige Krankheiten, deren Verlauf so entsetzliche Auswirkungen zeigte, dafür müsse man aber dort unter einer Durchseuchung des Geistes leiden, einer inneren, unheilbaren Morschheit, deren zersetzende Kraft sich wie ein Krebsgeschwür durch die Seele zu fressen vermochte. ${ }^{22}$

Das Fieber wird zu einer Achse, an der Engelhardt den Rat eines indischen Fakirs erproben kann, den er in Bombay erteilt bekommen hatte: „look at the world from different positions“. ${ }^{23}$ Die unterschiedlichen Positionen bedeuteten, den Eurozent-

\footnotetext{
${ }^{20}$ Die lange Liste der Publikationen im Fachbereich „Kolonialismus und Literatur“ aus den letzten Jahrzehnten unterstreicht das stets wachsende Interesse an den Kolonialexpeditionen. Einige wichtige Werke seien hier genannt: Thomas Schwarz: Robert Müllers Tropen. Heidelberg 2006; Dirk Göttsche: Remembering Africa. The Rediscovery of Colonialism in Contemporary German Literature. Rochester 2013; Axel Dunker: Kontrapunktische Lektüren. Koloniale Strukturen in der deutschsprachigen Literatur des 19. Jahrhunderts. München 2007; Alexander Honold, Klaus Scherpe (Hrsg.): Mit Deutschland um die Welt. Eine Kulturgeschichte des Fremden in der Kolonialzeit. Stuttgart/Weimar 2004.

${ }^{21}$ Fabian: Im Tropenfieber, S. 94.

${ }^{22}$ Kracht: Imperium, S. 70.

${ }^{23}$ Buhl: Das Paradies des August Engelhardt, S. 10
} 
rismus wie ein Gewand abzulegen und durch die Nacktheit eine durch Herrschaftssucht und Größenwahn unbelastete Perspektive anzunehmen. Engelhardt benutzt zwar in seinen Auseinandersetzungen mit Pater Joseph und Gouverneur Hahl das Malariafieber als das Pendant für die Krankheiten der Konsumsucht und des oberflächlichen Materialismus in der deutschen Gesellschaft, bleibt aber in seinem Profil des Pflanzers und Wirtschafters im kolonialen Rahmen sehr ambivalent. Einerseits tadelt er Pater Joseph, dass es den Eingeborenen nicht gelingt, in Frieden, Eintracht und Güte zu leben, weil die Missionare diese ,mit Religion und Moral, mit Arbeit und Steuern, mit den Segnungen einer Zivilisation [quälen], die selber verdammt ist $\mathrm{zu}$ sterben ${ }^{\text {‘2 } 24}$, andererseits will er auf wirtschaftliche Gewinne nicht verzichten. Es mag die Intention von Buhl gewesen sein, seinem Protagonisten eine europa-, missions-, und kolonialismuskritische Stimme zu verleihen, aber es gelingt ihm nicht ganz. Der Sonnenverehrer und Kokovore bleibt „eine Mischung aus Robinson, Winnetou, Lederstrumph und Rousseau“". ${ }^{25}$

Die Fieberträume, unter denen Engelhardt selbst leidet, als er an Scharlach erkrankt, sind Zeichen einer sehr effektiv eingesetzten Erzählstrategie von Buhl. Es sind Exkursionen in die Vergangenheit des Protagonisten, in der er seine verdrängten Liebesgefühle für Anna Schwab delirierend wieder erleben kann. Seine Ansicht, dass ,die Krankheit eine Reinigung des Körpers sei, der das Fieber und die Entzün-

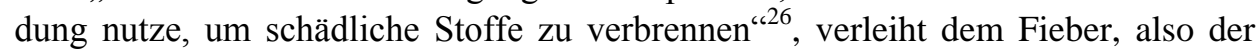
Krankheit selbst, eine heilende Funktion. So kommt hier die Dialektik des Fieberdiskurses zum Ausdruck. Die heißen Temperaturen dienen so den aufgewerteten Wunschvorstellungen der Reinigung und Erlösung, indes die „Zuvielisation“ ${ }^{27}$ in Deutschland und die Übersättigung, in die die ,Kathedralen des Konsums' (etwa das KDW) die Menschen treiben, als ,kalte' Abwertungsbilder gelten. Interessant ist die Zusammenführung der konsumkritischen Begriffe mit den religiösen Anstalten der vermeintlichen Reinigung des Menschen. So denunziert der Protagonist den „Dreiklang des zwanzigsten Jahrhunderts“, verkörpert durch „Geld, Gott und Dreck“. ${ }^{28}$

Was der sonnenfanatische Romanheld bei Buhl und Kracht durch seine Nacktheit versucht, wird bei Ilija Trojanow durch die Verkleidung als eine Assimilationsbzw. Aneignungstaktik ausgeübt. Der Indien-Teil seines Romans Der Weltensammler ist kein Versuch, ein Indienbild zu vermitteln. Im Gegenteil: Es geht um die Dialektik zwischen der ,An-eig-nung' (des Fremden durch das Eigene) und der ,Enteign-ung`/,Ent-selbst-ung` des Eigenen (Ich) durch das Fremde (Du). Gleich nach der Ankunft in Bombay muss sich der Protagonist, Richard Francis Burton, den

\footnotetext{
${ }^{24}$ Ebd., S. 23.

${ }^{25}$ Ebd., S. 30.

${ }^{26}$ Ebd., S. 42.

${ }^{27}$ Nach Marc Buhl stammt die Wortschöpfung von dem Künstler Diefenbach.

${ }^{28}$ Buhl: Das Paradies des August Engelhardt, S. 11.
} 
,Warnschildern' seiner Landsleute aussetzen. Alles warnt ihn vor seiner aktuellen Karrierestation namens Indien:

Hören Sie, junger Mann! [...] Das Klima: fatal, die Bediensteten: beschränkt, die Straßen: septisch, und die indischen Frauen: alles zugleich, weswegen diese, hören Sie gut zu, junger Mann!, unbedingt zu meiden sind [...]. Am besten - einen ehrlicheren Ratschlag werden Sie nicht zu hören bekommen - am besten, Sie halten sich von allem Fremden fern! $!^{29}$

Lahiya, der Schreiber in Weltensammler, und Kabua, der Tolai Häuptling in der Geschichte des August Engelhardt, erfüllen die Funktion eines Chronisten, aber sie problematisieren auch das Verhältnis von Fakten und Fiktion. Stefanie Catani nennt diese Art des Romans „,metafiktional“ ${ }^{30}$ Das Verhaltensmuster Burtons, seine Reaktionen auf seine Umwelt und deren Bewohner, seine Vorstellungen der Selbst- und Fremderfahrung, sind durch sichtbare Ambivalenzen hervorgehoben. Diese Ambivalenz lässt sich als eine „unvermeidbare Bedrohung“ erörtern, die, um mit Homi Bhabha zu sprechen, „von dem kulturell Anderen ausgeht, z. B. mit dem Gefühl von Fremdheit, von Andersartigkeit und fehlender Zugehörigkeit.“' ${ }^{31}$ Das trifft in vergleichbarem Maße auf die Figur Engelhardts zu.

Im Roman von Buhl werden verschiedene Formen der ,An-eig-nung', der „appropriation“ - in denen das Wort ,Eigen-tum' bzw. ,property' bereits enthalten ist - durchgespielt: Eine Variante ist eine militärische Eroberung und Landvermessung, die das ,An-ge-eig-nete' zerstört; daneben steht die religiöse Missionierung, die höhere Formen des Glaubens und der Welterklärung ersetzen will; schließlich geht es um die Aufpfropfung eines fremden Rechtssystems - alles gewalttätige Weisen der ,An-eig-nung'. Konkrete Beispiele sind die Strafexpeditionen im Kolonialreich oder ein Versuch der Verwaltungsherren, „die Eingeborenen in die deutsche, aufrichtig moralische Gesetzbarkeit einzubinden “32. Hier wird auch eine mit anderen Kolonialmächten Europas konkurrierende Überlegenheit der Deutschen bemerkbar. Die Deutschen haben ihre Verwaltungsmodi in ihrem Kolonialreich für eine „hochsittliche, faire Instanz ${ }^{633}$ gehalten und dabei die Franzosen, Engländer, Niederländer und Belgier für ihre ausbeuterischen Praktiken der modernen Sklaverei denunziert. Die vergleichende Betrachtung des Indien-Romans Trojanows mit den DeutschNeuguinea Romanen von Buhl und Kracht zeigt, dass der Kolonialismus-Diskurs

\footnotetext{
${ }^{29}$ Trojanow: Der Weltensammler, S. 23.

${ }^{30}$ Stephanie Catani: Metafiktionale Geschichte(n). Zum unzuverlässigen Erzählen historischer Stoffe in der Gegenwartsliteratur. In: Christof Hamann, Alexander Honold (Hrsg.): Ins Fremde schreiben. Gegenwartsliteratur auf den Spuren historischer und fantastischer Entdeckungsreisen. Göttingen 2009, S. 143-168, hier S. 154.

${ }^{31}$ Homi Bhabha: Die Verortung der Kultur. Übersetzt von Michael Schiffmann und Jürgen Freundl. Tübingen 2000, S. X.

${ }^{32}$ Buhl: Das Paradies des August Engelhardt, S. 169.

${ }^{33}$ Ebd.
} 
sich nicht auf bestimmte Länder und deren konkrete Kolonien beschränken lässt. Richard Francis Burton lernt indische Sprachen, schläft mit indischen Kurtisanen, isst und trinkt wie die Inder. Das sind für seine Landsleute alternative Wege der ,An-eig-nung ‘. Diese Versuche setzen jedoch eine gewisse Distanz zur eigenen Herkunft voraus: „Er hat bei den Seinen den Ruf eines Unberührbaren““34 und „[e]r hatte nur wenig übrig für den Glauben seiner Leute, die eigenen Bräuche seien für ihn nur Aberglauben, die fremden Bräuche hingegen seien faszinierend“، 35

Vor dem Eindringen in die metaphysische Dimension des Fremden steht die physische Beschneidung am eigenen Körper, dem Glaubenswechsel (Burton konvertiert zum Islam) geht wiederum ein Ortswechsel voraus. Burtons Verkleidungspraktiken und Versuche, dem Fremden näher zu kommen, sowie Engelhardts alternative, lebensreformierende Ideen bzw. Kokovorismus, Sonnenverehrung, Nacktheit, Entsagung der materiellen Besitztümer etc. münden schließlich in den gleichen Strom der Ich-Bezogenheit. Burtons Weg zur Assimilation geht zunächst über das noch funktional motivierte Erlernen der indischen Hochsprachen (,Ich will dem Ennui entkommen, indem ich lerne ${ }^{\text {(36) }}$, die auch seine Karriere befördern können. Dies ist eine Form der geistigen ,Ein-ver-leib-ung', die ebenso die rigorose Verbannung der eigenen Sprache (,Ent-eig-nung' des Eigenen als des für die Anderen Fremden) aus dem täglichen Umfeld erzwingt. ${ }^{37}$ Schließlich muss die ,An-eig-nung ' Burton auf den Leib geschrieben sein - daher seine Neigung zur Verkleidung, Maskerade und Camouflage durch diverse Accessoires. Er betrachtet die Kleidung als die äußere, die Sprache hingegen als die innere Schicht der Identität - zum nachgeahmten Zungenschlag kommt die richtige Kopfbedeckung. ${ }^{38}$ Bei der ,An-eig-nung' und ,Einver-leib-ung' geht es eben nicht nur um eine geistige Auseinandersetzung, sondern auch um existentielle Akte.

Das bedeutet aber, dass das Ziel einer solchen alternativen Appropriation der fremden Kultur deren Bewahren (conservatio) ist und nicht deren Veränderung: Veränderung bedeutet im kulturellen Bereich eine Elimination, ein Auslöschen, was bei Trojanow die Mission des Generals, bei Buhl und Kracht die Missionen von Pater Joseph und von Gouverneur Hahl vor Augen führen. Die alternative Form der ,An-eig-nung' ist somit konservativ, sie sieht bereits in der nivellierenden Veränderung - z. B. durch die Einführung angeblich universeller Menschenrechte - keinen Fortschritt, keine Progression, sondern eine Regression. Leutnant Burton ist mit seinen Versuchen der Assimilation für die britische Ostindiengesellschaft, und besonders für deren militärischen Arm, nicht akzeptabel. Aus demselben Grund wird Engelhardt zu einem Störfaktor für die deutschen Kolonialherren.

\footnotetext{
${ }^{34}$ Trojanow: Der Weltensammler, S. 58.

${ }^{35}$ Ebd., S. 63.

${ }^{36}$ Ebd., S. 59

${ }^{37}$ Ebd., S. 53.

${ }^{38}$ Vgl. ebd., S. 80.
} 
Hansjörg Bay diskutiert die historischen und wissenschaftlichen Bezüge der Bedürfnisse und Auswirkungen solcher Assimilationsversuche im Rahmen des Kolonialdiskurses sowie der Ethnographie. Er weist auf den Ausdruck, Verkafferung ' aus dem Deutschen Koloniallexikon hin, in dem jeder Aneignungsversuch eines Europäers, sich auf die Gewohnheiten und den Lebensstil der Einheimischen einzulassen, als eine „bedauerliche Entartung des weißen Ansiedlers“ ${ }^{\text {(39 }}$ betrachtet wurde. Die Kolonialbürokratie warnte ihre Soldaten, Offiziere, Verwaltungsbeamten und Missionare, sich an die Spielregeln zu halten, d. h. sich vor jeder Annäherung zu hüten. Manche nannten es „falsche Wißbegier“40 und für manche war ,jedes Überschreiten der Grenze und jedes Verwischen der Unterschiede zu den Kolonisierten “41 einfach inakzeptabel. Im Roman von Trojanov erfüllen der Sanitäter und andere Engländer diese Warnschildfunktion, indem sie Burton gegenüber Indien wie eine ansteckende Krankheit darstellen. In den Romanen von Buhl und Kracht sind es Verwaltungsangestellte, Missionare oder weiße Frauen, die sich vor einem nackten Landsmann schämen müssen. Bay perspektiviert das Phänomen der Assimilation und stellt fest, dass der koloniale Begriff der 'Verkafferung' als Synonym zu dem in der Ethnographie gebräuchlichen, going native' zu denken ist.

Der Temperaturendiskurs geht in den analysierten Werken mehrere Schleifwege. Die heißen Temperaturen fungieren in binärer Weise - einerseits hat die Hitze eine heilende Funktion und andererseits löst sie Unheil aus. Auf einmal werden die Temperaturen mehr als nur eine meteorologische Einheit. Das Adjektiv ,heiß`symbolisiert gleichzeitig das Kolonisierte, das Primitive, das ,edle' und zugleich das ,unedle“ Wilde. Die Konstellationen Tropen, Temperaturen, Körper und Kolonialismus lassen sich immer neu und immer anders konfigurieren, aber die Verbindung bleibt unübersehbar.

\section{Literatur}

Achebe, Chinua: Ein Bild von Afrika. Rassismus in Conrads „Herz der Finsternis“. Deutsch von Thomas Brückner und Wulf Teichmann. Berlin 2000.

Bay, Hansjörg: Going Native? Mimicry und Maskerade in kolonialen Entdeckungsreisen der Gegenwartsliteratur. In: Christof Hamann, Alexander Honold (Hrsg.): Ins Fremde schreiben. Gegenwartsliteratur auf den Spuren historischer und fantastischer Entdeckungsreisen. Göttingen 2009, S. 117-142.

Bhabha, Homi: Die Verortung der Kultur. Übersetzt von Michael Schiffmann und Jürgen Freundl. Tübingen 2000.

\footnotetext{
${ }^{39}$ Hansjörg Bay: Going Native? Mimicry und Maskerade in kolonialen Entdeckungsreisen der Gegenwartsliteratur. In: Hamann, Honold (Hrsg.): Ins Fremde schreiben, S. 117-142, hier S. 117.

${ }^{40}$ Trojanow: Der Weltensammler, S. 22.

${ }^{41}$ Bay: Going Native?, S. 118.
} 
Buhl, Marc: Das Paradies des August Engelhardt. Roman. Frankfurt a. M. 2011.

Catani, Stephanie: Metafiktionale Geschichte(n). Zum unzuverlässigen Erzählen historischer Stoffe in der Gegenwartsliteratur. In: Christof Hamann, Alexander Honold (Hrsg.): Ins Fremde schreiben. Gegenwartsliteratur auf den Spuren historischer und fantastischer Entdeckungsreisen. Göttingen 2009, S. 143-168.

Dunker, Axel: Kontrapunktische Lektüren. Koloniale Strukturen in der deutschsprachigen Literatur des 19. Jahrhunderts. München 2007.

Dürbeck, Gabriele: Stereotype Paradiese. Ozeanismus in der deutschen Südseeliteratur. 1815-1914. Tübingen 2007.

Fabian, Johannes: Im Tropenfieber. Wissenschaft und Wahn in der Erforschung Zentralafrikas. München 2001.

Göttsche, Dirk: Remembering Africa. The Rediscovery of Colonialism in Contemporary German Literature. Rochester 2013.

Honold, Alexander, Klaus Scherpe (Hrsg.): Mit Deutschland um die Welt. Eine Kulturgeschichte des Fremden in der Kolonialzeit. Stuttgart/Weimar 2004.

Janz, Rolf-Peter (Hrsg.): Faszination und Schrecken des Fremden. Frankfurt a. M. 2001.

Kracht, Christian: Imperium. Roman. Köln 2012.

Lützeler, Paul Michael (Hrsg.): Der postkoloniale Blick. Deutsche Schriftsteller berichten aus der Dritten Welt. Frankfurt a. M. 1997.

Repussard, Catherine: Ein bisschen Südsee und ein gutes Maß Lebensreform: Das Rezept für das beginnende 21. Jahrhundert? Marc Buhls „Paradies des August Engelhardt“ (2011) und Christian Krachts „Imperium “ (2012). In: Recherches Germaniques 42 (2012), S. 77-98.

Schwarz, Thomas: Robert Müllers Tropen. Heidelberg 2006.

Trojanow, Ilija: Der Weltensammler. Roman. München 2006.

Uerlings, Herbert: Verkehrte Welten. Primitivismus in Literatur und Kunst der Klassischen Moderne. In: Tobias L. Kienlin (Hrsg.): Fremdheit - Perspektiven auf das Andere. Bonn 2015 (= Kölner Beiträge zu Archäologie und Kulturwissenschaften, Bd. 264), S. 9-24. 